\title{
Palladium/Norbornene Chemistry in the Synthesis of Polycyclic Indolines with Simple Nitrogen Sources
}

\author{
Mehran Ghasemia,b \\ Farnaz Jafarpour*a \\ Azizollah Habibi*b \\ a School of Chemistry, College of Science, University of Tehran, \\ 14155-6455 Tehran, Iran \\ jafarpur@khayam.ut.ac.ir \\ b Faculty of Chemistry, Kharazmi University, No. 43 Mofateh \\ Street, Enghelab Ave., 15719-14911 Tehran, Iran \\ habibi@khu.ac.ir
}

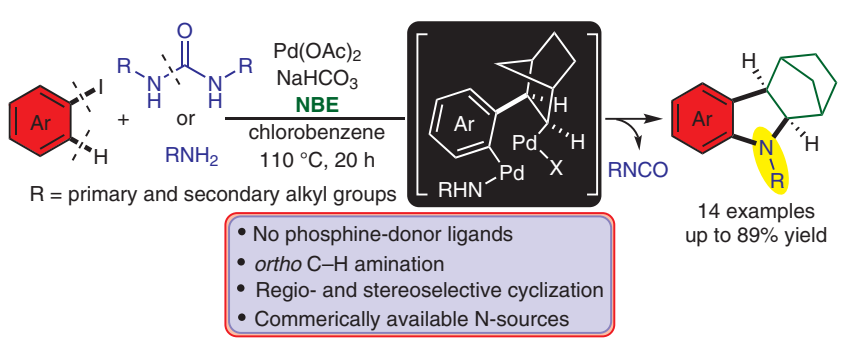

Received: 19.12.2019

Accepted after revision: 15.02 .2020

Published online: 27.03 .2020

DOI: 10.1055/s-0039-1707988; Art ID: ss-2019-t0694-op

Abstract An efficient procedure has been developed to synthesize indoline derivatives through a palladium-catalyzed Heck reaction/C-H activation/dual amination cascade in one pot. This constitutes the first intermolecular catalytic approach to directly access $\mathrm{N}$-alkylindolines with a broad substrate scope in the absence of any ligands. This method highlights the use of readily available amines and ureas as the required nitrogen sources in building up the indoline core.

Key words amines, $\mathrm{N}$-alkylindolines, norbornene, palladacycle, urea

The synthesis of heterocycles using various methods has been of great interest in recent decades. Indolines are one of the heterocycles that have undergone various synthetic methods in recent years. ${ }^{1}$ The main reason for focusing on the synthesis of structures containing indoline scaffold is the unique biological and pharmacological properties of these compounds. ${ }^{2}$ The structures with indoline skeletons are ubiquitously present in many naturally bioactive alkaloids, such as strychnine, ${ }^{3}(-)$-physostigmine, ${ }^{4}$ and (+)-aspidospermidine $^{5}$ (Figure 1 ). It is also a vital intermediate of the pentopril, a drug used for the treatment of hypertension (Figure 1). ${ }^{6}$ Recently, Du and co-workers have also isolated oleracein from the edible plant Portulaca oleracea used in Chinese traditional medicine (Figure 1$).{ }^{7}$ Given the importance of these structures, the synthesis of indoline derivatives has been a research topic of great interest to research chemists since the last decade.

One of the most important methods for the construction of indoline scaffolds is the intramolecular BuchwaldHartwig amination reaction of amine-tethered aryl halides (Scheme 1a). ${ }^{8}$ Recently, Yu et al..$^{9 a}$ pioneered an alternative auxiliary directed indoline synthesis through aryl $\mathrm{C}-\mathrm{H}$ acti-

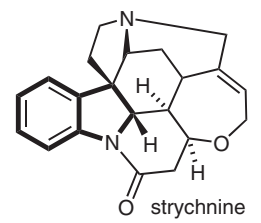<smiles>CNC(=O)Oc1ccc2c(c1)C1(C)CCN(C)C1N2[14CH3]</smiles><smiles>CCOC(=O)C[C@H](C)C(=O)N1c2ccccc2C[C@H]1C(=O)O</smiles>

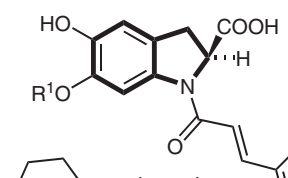<smiles>[R7]C1=C([R7])CCC(C)=C1</smiles>

(+)-aspidospermidine

Figure 1 Representative bioactive indolines and its derivatives

vation/intramolecular amination process, which was further improved by employing various $\mathrm{N}$-chelating groups and oxidizing agents such as hypervalent iodonium salts (Scheme 1b). ${ }^{9}$ While these methods provide an attractive entry to these ring systems, they are mostly limited to intramolecular amination reactions and rely on the use of substrates that are preinstalled with amino groups. Furthermore, Kapur et al. communicated a palladium-catalyzed intramolecular $\alpha$-arylation of silyl enol ethers of $\beta$ aminoketones, which led to the formation of the 3-substituted indolines (Scheme 1c). ${ }^{10}$ However, the multi-step reaction and limitation of product diversity were some drawbacks of the report. Glorius et al. also developed a Rh(III)catalyzed directed $\mathrm{C}-\mathrm{H}$ activation, followed by intramolecular addition of the $\mathrm{Csp}^{3}-\mathrm{Rh}$ species to the $\mathrm{N}=\mathrm{N}$ bond to afford 1-aminoindolines using Boc-protected aryldiazenes and alkenes (Scheme 1d). ${ }^{11}$ 


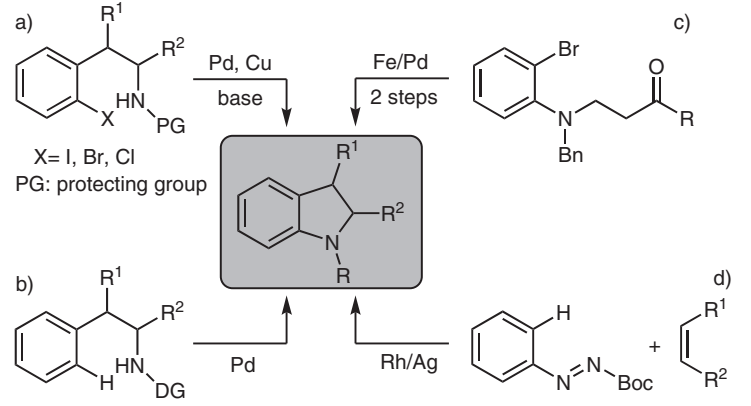

$D G$ : directing group

Scheme 1 Transition-metal-catalyzed synthesis of indolines

In the last decade, the strategy of using norbornene for activation of $\mathrm{C}-\mathrm{H}$ bonds has been used to synthesize different heterocycles. This technique benefits from the high reactivity of norbornene in the activation of the inactive ortho $\mathrm{C}-\mathrm{H}$ bonds in aryl halides and palladacycle formation.

This strategy was devised by Catellani and further developed by other research groups. ${ }^{12}$ Recently the application of palladium/norbornene (Pd/NBE) chemistry for construction of indolines via amination of aryl-norbornenepalladacycle (ANP) intermediates employing three membered strained $\mathrm{N}$-containing heterocycles have become realistic (Scheme 2). The Shi group pioneered the construction of indolines via oxidative addition of the C,C-palladacycle to di-tert-butyldiaziridinone (Scheme $2 \mathrm{a}$ ). ${ }^{13}$

Furthermore, Bi and Liang extended the scope of $\mathrm{Pd} / \mathrm{NBE}$ chemistry employing sulfonated aziridines as electrophilic reagents for ortho-amination of iodoarenes and construction of $\mathrm{N}$-tosylindolines (Scheme $2 \mathrm{~b}$ ). ${ }^{14}$

Despite the importance of these communications, the protocols showed a narrow substrate scope while the products were limited to $\mathrm{N}-\mathrm{t} \mathrm{Bu}$ and $\mathrm{N}$-Ts indolines, as well as cost issues linked to the use of strained three-membered $\mathrm{N}$ heterocyclic rings. The last report in this context belongs to Dai and Hu who established a decarboxylative annulation of 2-haloaroyloxycarbamates with norbornene for the construction of indolines (Scheme $2 \mathrm{c}$ ). ${ }^{15}$ This protocol encountered similar scope limitations on the N-substituent of indolines and required prefunctionalized starting materials. We also recently reported on a regioselective annulation reaction to provide $\mathrm{N}$-arylindolines as a new outcome from the palladium-catalyzed reaction of iodoarenes, norbornene, and anilines. ${ }^{16}$ Despite significant achievements, however, aliphatic amines remained challenging and more difficult than aromatic amines for this catalytic system. Aliphatic ureas also did not participate in this cascade. Considering the high importance of indoline scaffolds in pharmaceutics and remarkable effect of the nature of the N-substituent of $\mathrm{N}$-heterocycles on their biological properties, it would be highly desirable to directly construct complicated indoline molecules from simple and readily available starting materials and more easily diversified nitrogen sources. a)

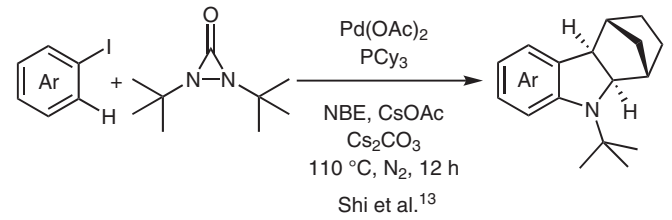

b)

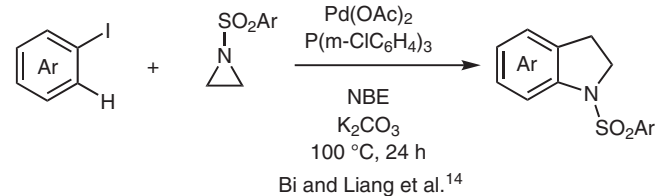

c)<smiles>O=C(NOC(=O)c1ccccc1I)c1ccccc1</smiles><smiles>O=C(OCc1ccccc1)OC(=O)N1c2ccccc2[C@H]2[C@H]3CC[C@H](C3)[C@H]21</smiles>

this work:

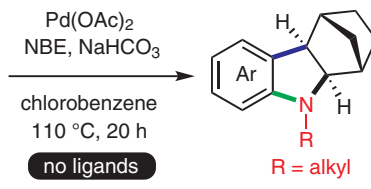

ortho-amination reaction

readily available nitrogen sour no phosphine-donor ligands
highly regioselective

highly regioselective

Scheme 2 Pd/NBE chemistry in the synthesis of indolines

Herein we report an effective method for the synthesis of various polycyclic indolines via Pd/NBE chemistry employing three readily available and easily diversified building blocks including: iodoarenes, norbornene, and readily available nitrogen sources including aliphatic amines and ureas (Scheme 2d). This protocol has the potential for construction of indoline motifs easily diversified on both arene and nitrogen sides, which has not been accomplished yet. Setting the competing ipso-amination versus ortho-amination in the presence of nucleophilic amine sources is the key step for this transformation. This reaction would offer distinct advantages over existing methods, particularly with respect to functional group compatibility on both arene and nitrogen sides, accessible and economical nitrogen sources, and excluding any requisite for phosphinedonor ligands usually necessary in Pd-catalyzed reactions.

In a typical experiment, 2-iodotoluene (1a), propylamine (2a), and norbornene were reacted in the presence of $\mathrm{PdCl}_{2}, \mathrm{PPh}_{3}, \mathrm{Cs}_{2} \mathrm{CO}_{3}$ in $\mathrm{MeCN}$ at $110{ }^{\circ} \mathrm{C}$ for 20 hours. Under these conditions, polyclic indoline 3a was fortunately collected in $21 \%$ yield (Table 1 , entry 1 ). The reaction was optimized with respect to Pd sources where $\mathrm{Pd}(\mathrm{OAc})_{2}$ proved to be the most effective catalytic system (entries 1-3). Employing different bases, sodium bicarbonate exhibited the best performance in the annulation reaction (entries 4-7). 
However, conducting the reaction in the presence of a stronger base such as $\mathrm{NaOH}$ led to a decrease in the yield (entry 6). Gratefully, the transformation proceeded well in the absence of any added ligands rarely achieved in similar methodologies (entry 8). Therefore, the optimization of reaction was continued without using any additional ligands. Next, the effect of different solvents on this transformation was investigated (entries 8-13). Screening of the solvents showed that chlorobenzene was the best choice of solvent and gave the desired product 3a in 69\% isolated yield (entry 13). Increasing the amount of norbornene to 4 equivalents did not improve the reaction yield (entry 14). Finally, reducing temperature to $90^{\circ} \mathrm{C}$ led to a yield bargain (entry 15).

Table 1 Optimization of Reaction Conditions for the Annulation of 2-Iodotoluene ${ }^{\text {a }}$

\begin{tabular}{|c|c|c|c|c|c|}
\hline Entry & Catalyst & L & Base & Solvent & Yield (\%) \\
\hline 1 & $\mathrm{PdCl}_{2}$ & $\mathrm{PPh}_{3}$ & $\mathrm{Cs}_{2} \mathrm{CO}_{3}$ & $\mathrm{MeCN}$ & 21 \\
\hline 2 & $\mathrm{Pd}(\mathrm{OAc})_{2}$ & $\mathrm{PPh}_{3}$ & $\mathrm{Cs}_{2} \mathrm{CO}_{3}$ & $\mathrm{MeCN}$ & 38 \\
\hline 3 & $\mathrm{Pd}(\mathrm{dba})_{2}$ & $\mathrm{PPh}_{3}$ & $\mathrm{Cs}_{2} \mathrm{CO}_{3}$ & $\mathrm{MeCN}$ & 19 \\
\hline 4 & $\mathrm{Pd}(\mathrm{OAC})_{2}$ & $\mathrm{PPh}_{3}$ & $\mathrm{~K}_{2} \mathrm{CO}_{3}$ & $\mathrm{MeCN}$ & 37 \\
\hline 5 & $\mathrm{Pd}(\mathrm{OAc})_{2}$ & $\mathrm{PPh}_{3}$ & $\mathrm{~K}_{3} \mathrm{PO}_{4}$ & $\mathrm{MeCN}$ & 42 \\
\hline 6 & $\mathrm{Pd}(\mathrm{OAc})_{2}$ & $\mathrm{PPh}_{3}$ & $\mathrm{NaOH}$ & $\mathrm{MeCN}$ & 13 \\
\hline 7 & $\mathrm{Pd}(\mathrm{OAc})_{2}$ & $\mathrm{PPh}_{3}$ & $\mathrm{NaHCO}_{3}$ & $\mathrm{MeCN}$ & 52 \\
\hline 8 & $\mathrm{Pd}(\mathrm{OAc})_{2}$ & - & $\mathrm{NaHCO}_{3}$ & $\mathrm{MeCN}$ & 54 \\
\hline 9 & $\mathrm{Pd}(\mathrm{OAC})_{2}$ & - & $\mathrm{NaHCO}_{3}$ & toluene & 28 \\
\hline 10 & $\mathrm{Pd}(\mathrm{OAC})_{2}$ & - & $\mathrm{NaHCO}_{3}$ & THF & trace \\
\hline 11 & $\mathrm{Pd}(\mathrm{OAC})_{2}$ & - & $\mathrm{NaHCO}_{3}$ & DMF & 37 \\
\hline 12 & $\mathrm{Pd}(\mathrm{OAc})_{2}$ & - & $\mathrm{NaHCO}_{3}$ & DMSO & 41 \\
\hline 13 & $\operatorname{Pd}(\mathrm{OAC})_{2}$ & - & $\mathrm{NaHCO}_{3}$ & chlorobenzene & 69 \\
\hline $14^{\mathrm{b}}$ & $\mathrm{Pd}(\mathrm{OAc})_{2}$ & - & $\mathrm{NaHCO}_{3}$ & chlorobenzene & 65 \\
\hline $15^{c}$ & $\mathrm{Pd}(\mathrm{OAC})_{2}$ & - & $\mathrm{NaHCO}_{3}$ & chlorobenzene & 55 \\
\hline
\end{tabular}

${ }^{a}$ Reaction conditions: 2 -iodotoluene ( $1 \mathbf{a} ; 0.1 \mathrm{mmol}$ ), norbornene ( 2 equiv), propylamine (2a; 2 equiv), catalyst ( $5 \mathrm{~mol} \%$ ), ligand ( $10 \mathrm{~mol} \%)$, base (2 equiv), and solvent $\left(1 \mathrm{~mL}\right.$ ) at $110^{\circ} \mathrm{C}$ for $20 \mathrm{~h}$.

b Norbornene: 4 equiv.

C Reaction temperature: $90^{\circ} \mathrm{C}$.

With the optimized condition in hand, the scope of the annulation reaction to construct various polycyclic indolines was examined. As summarized in Scheme 3, a wide range of substituted iodoarenes and amines were found to be compatible with this domino Heck reaction/double C-N bond formation.

First the reactivity of 2-iodotoluene with various alkyl amines was examined and yields were obtained between 57-88\%. Fortunately, palladium-catalyzed annulation of iodoarenes and some bulkier amines such as isopropylamine and cyclohexylamine proceeded smoothly under the optimized reaction conditions to afford the desired products $\mathbf{3 g}$
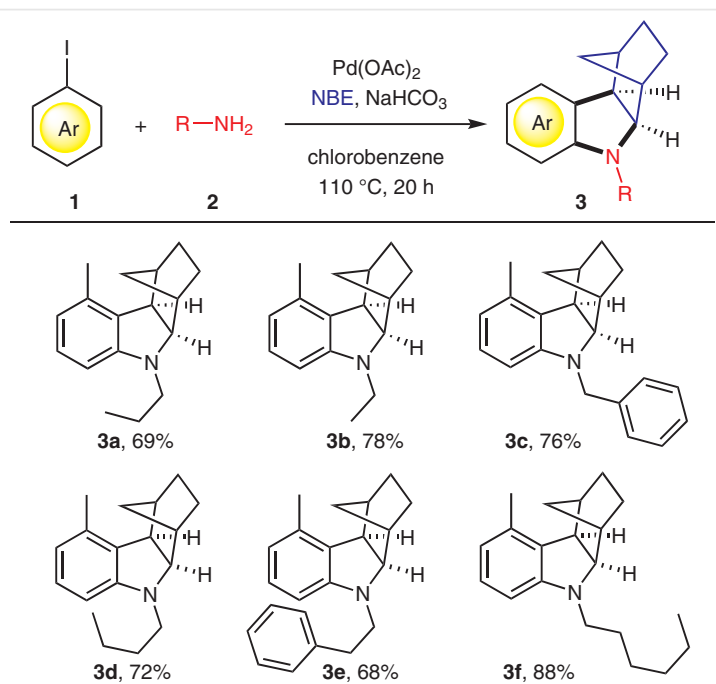

3d, $72 \%$

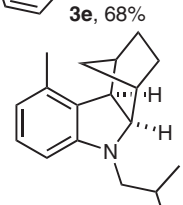

3f, $88 \%$<smiles>Cc1cccc2c1C1CCC(C1)[C@H]2N(C)C(C)C</smiles><smiles>CC1=C2C(=CCCC2C)N[C@H](C)[C@H]1C</smiles>

$3 g, 81 \%$ $3 \mathrm{~h}, 66 \%$

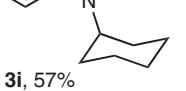<smiles>COCCN1c2cccc(C)c2C2(C)CCC(C2)[C@H]1C</smiles>

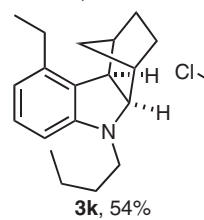
3i, $57 \%$
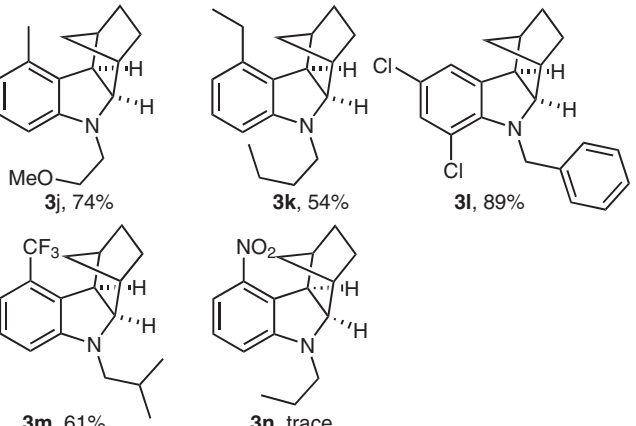

3I, $89 \%$

$3 n$, trace

Scheme 3 Reaction scope for construction of indolines

and $\mathbf{3 i}$ in $81 \%$ and $57 \%$ isolated yield, respectively. According to the biological importance of $\mathrm{N}$-cyclohexylindoline derivatives, the synthesis of these compounds has received great attention. ${ }^{17}$ Intriguingly, even iodoarene containing susceptible halo groups could be well tolerated under the reaction conditions to provide the desired product $\mathbf{3 1}$ in promising $89 \%$ isolated yield with preserved chloro groups. This adduct can serve as a good precursor for further functionalizations through metal-catalyzed cross-coupling reactions. In addition, the ortho-trifluoromethyl-substituted iodoarene, which due to its lower reactivity is relatively rarely used in palladium-catalyzed coupling reactions, was compatible with the current reaction conditions affording the desired polycyclic indoline $3 \mathrm{~m}$ in $61 \%$ isolated yield. Notably, yields of $58 \%$ and $71 \%$ were still obtained for compounds $\mathbf{3 a}$ and $\mathbf{3 c}$, respectively, when the reactions were scaled up to $4.0 \mathrm{mmol}$. Unfortunately, nitro-substituted iodoarene did not participate in this transformation. 
Gratefully, when expanding the scope of amines to their amide derivatives $\mathbf{4}$, we found that ureas could also serve as the nitrogen sources of indolines through $\mathrm{C}-\mathrm{N}$ bond cleavage. ${ }^{18}$ Once aliphatic amines were replaced with 1,3 -bis(alkyl)ureas the annulation reactions proceeded well under the same optimized reaction conditions to afford the polycyclic indolines in comparable yields (Scheme 4).

$$
\begin{aligned}
110^{\circ} \mathrm{C}, 20 \mathrm{~h} & \\
\mathbf{3 a}, \mathrm{R}=\text { propyl } & 74 \% \\
\mathbf{3 b}, \mathrm{R}=\text { ethyl } & 69 \% \\
\mathbf{3 g}, \mathrm{R}=\text { isopropyl } & 67 \% \\
\mathbf{3 h}, \mathrm{R}=\text { isobutyl } & 64 \% \\
\mathbf{3 i}, \mathrm{R}=\text { cyclohexyl } & 58 \%
\end{aligned}
$$

Scheme 4 Scope of ureas as efficient nitrogen sources in the construction of indolines

Remarkably, when an asymmetric $\mathrm{N}$-alkyl, $\mathrm{N}$-arylurea was picked as a coupling partner in this transformation, a high regioselectivity was observed in the conversion. It is interesting to note that in this catalytic system, selectivity favored $\mathrm{N}$-alkylation versus $\mathrm{N}$-arylation of $\mathrm{C}-\mathrm{H}$ bonds for construction of $N$-alkylindoline 3a in 59\% yield and phenyl isocyanate was removed from the reaction pot as the byproduct (Scheme 5).

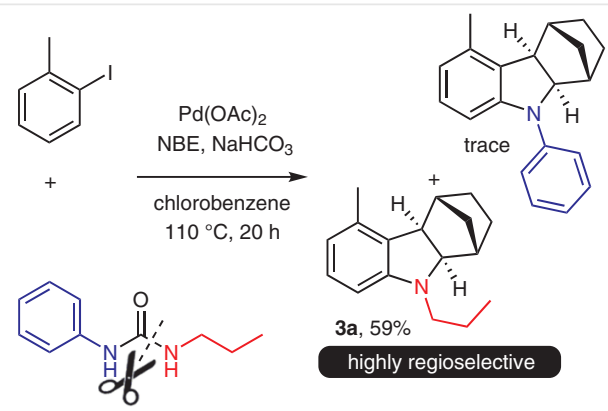

Scheme 5 Regioselectivity of the reaction with unsymmetrical ureas

A mechanistic rationale is summarized in Scheme 6. In the catalytic cycle, when amine is used as the coupling partner, the oxidative addition of aryl halide to $\operatorname{Pd}(0)$, followed by a carbopalladation reaction with NBE and subsequent intramolecular C-H activation, results in the C,C-palladacycle intermediate $\mathbf{5}$. Next a transmetalation between two $\mathrm{Pd}(\mathrm{II})$ centers, palladacycle $\mathbf{5}$ and $\mathrm{Pd}(\mathrm{II})$ coordinated to nitrogen, put forward by Cardenas and Echavarren ${ }^{19}$ and developed by Derat and Catellani, ${ }^{20}$ generates a binuclear $\mathrm{Pd}(\mathrm{II})$ intermediate 6.

After reductive elimination, $\operatorname{Pd}(0)$ is released and the first $\mathrm{C}-\mathrm{N}$ bond is forged (intermediate 7 ). Next the intramolecular coordination of nitrogen to the remaining Pd(II) cen-

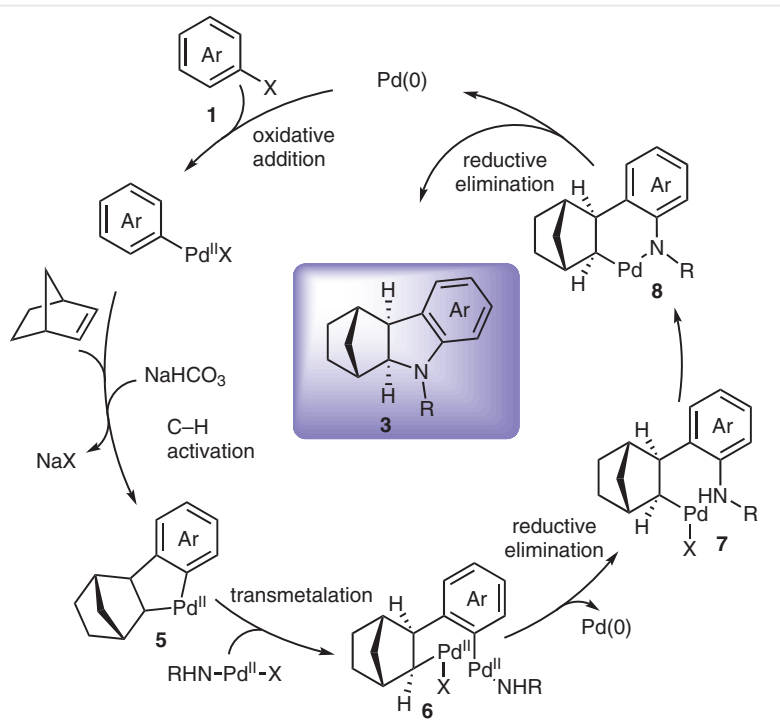

Scheme 6 Mechanistic rationale

ter forms intermediate $\mathbf{8}$, which on the second reductive elimination releases the next $\operatorname{Pd}(0)$ and installs nitrogen on two $\mathrm{Csp}^{3}$ and $\mathrm{Csp}^{2}$ bonds to give rise to polycyclic indoline 3.

In summary, we have developed an efficient method for construction of polycyclic indolines via amination of arylnorbornene-palladacycle as the key intermediate in Pd/NBE chemistry, employing readily available nitrogen sources such as aliphatic amines and ureas and building three $\mathrm{Csp}^{3}-$ $\mathrm{Csp}^{2} / \mathrm{Csp}^{3}-\mathrm{N} / \mathrm{Csp}^{2}-\mathrm{N}$ bonds in a single synthetic process. This approach provides a general platform to introduce various $N$-alkyl groups to the arene ortho-position and to provide various $\mathrm{N}$-alkyl-substituted indolines. The reaction features broad substrate scope and proceeds smoothly without any added phosphine-donor ligands usually as a prerequisite in palladium-catalyzed reactions. Employing unsymmetrical $\mathrm{N}$-alkyl, $\mathrm{N}$-arylurea, a high regioselectivity via ortho $\mathrm{N}$-alkylation versus $\mathrm{N}$-arylation of iodoarene was perceived.

All reagents were commercially available and used as received. Column chromatography was carried out on silica gel (230-400 mesh). ${ }^{1} \mathrm{H}$ NMR spectra were recorded at r.t. on a Bruker $500 \mathrm{MHz}$ spectrometer using DMSO- $d_{6}$ and $\mathrm{CDCl}_{3}$ as solvent. Chemical shifts are reported in ppm with TMS as an internal standard. ${ }^{13} \mathrm{C}$ NMR spectra are referenced from the solvent central peak. Chemical shifts are given in ppm. Elemental analyses (CHN) were recorded on a Thermo Finnigan Flash EA 1112 elemental analyzer.

5-Methyl-9-propyl-2,3,4,4a,9,9a-hexahydro-1H-1,4-methanocarbazole (3a); Typical Procedure

A vial equipped with a stir bar was charged with 2-iodotoluene (1a; $21.8 \mathrm{mg}, 0.10 \mathrm{mmol}$ ), propylamine (2a; $11.8 \mathrm{mg}, 0.2 \mathrm{mmol}, 2$ equiv), $\mathrm{Pd}(\mathrm{OAc})_{2}$ (1.1 mg, $5 \mathrm{~mol} \%$ ), norbornene ( $18.8 \mathrm{mg}, 0.2 \mathrm{mmol}, 2$ equiv), 
$\mathrm{NaHCO}_{3}$ (16.8 mg, $0.2 \mathrm{mmol}, 2$ equiv), and chlorobenzene $(1 \mathrm{~mL})$ was added, and the vial was capped. The resulting mixture was heated in a sand bath at $110^{\circ} \mathrm{C}$ for $20 \mathrm{~h}$, cooled, then filtered through a short plug of silica gel. Removal of the solvent gave a crude mixture, which was purified by column chromatography (hexane/EtOAc gradient) to give indoline 3a; yield: $17 \mathrm{mg}$ (69\%); yellow oil.

${ }^{1} \mathrm{H}$ NMR $\left(500 \mathrm{MHz}, \mathrm{CDCl}_{3}\right): \delta=0.96(\mathrm{t}, J=7.4 \mathrm{~Hz}, 3 \mathrm{H}), 1.14-1.23(\mathrm{~m}, 2$ H), 1.33-1.39 (m, $1 \mathrm{H}), 1.53-1.67(\mathrm{~m}, 5 \mathrm{H}), 2.24(\mathrm{~s}, 3 \mathrm{H}), 2.37(\mathrm{~s}, 1 \mathrm{H})$, $2.41(\mathrm{~s}, 1 \mathrm{H}), 3.03-3.15(\mathrm{~m}, 2 \mathrm{H}), 3.21(\mathrm{~d}, J=8.3 \mathrm{~Hz}, 1 \mathrm{H}), 3.63(\mathrm{~d}, J=8.3$ $\mathrm{Hz}, 1 \mathrm{H}), 6.12(\mathrm{~d}, J=7.1 \mathrm{~Hz}, 1 \mathrm{H}), 6.35(\mathrm{~d}, J=7.5 \mathrm{~Hz}, 1 \mathrm{H}), 6.94(\mathrm{t}, J=$ $7.65 \mathrm{~Hz}, 1 \mathrm{H})$.

${ }^{13} \mathrm{C}$ NMR $\left(125 \mathrm{MHz}, \mathrm{CDCl}_{3}\right): \delta=11.7,18.4,21.0,25.0,29.0,32.7,41.0$, 41.4, 49.4, 50.3, 71.9, 102.1, 117.3, 127.7, 129.6, 133.9, 153.6.

EI-MS: $m / z(\%)=241\left(\mathrm{M}^{+}, 100\right), 173(49), 198(27)$.

Anal. Calcd for $\mathrm{C}_{17} \mathrm{H}_{23} \mathrm{~N}$ : C, 84.59; H, 9.60; N, 5.80. Found: C, 84.93; H, $9.74 ; \mathrm{N}, 6.09$.

\section{5-Methyl-9-propyl-2,3,4,4a,9,9a-hexahydro-1H-1,4-methano- carbazole (3b)}

Yield: $18 \mathrm{mg}$ (78\%); yellow oil.

${ }^{1} \mathrm{H}$ NMR (500 MHz, $\left.\mathrm{CDCl}_{3}\right): \delta=0.89-0.92(\mathrm{~m}, 1 \mathrm{H}), 1.14(\mathrm{t}, J=6.9 \mathrm{~Hz}, 3$ H), $1.28(\mathrm{~s}, 3 \mathrm{H}), 1.52-1.59(\mathrm{~m}, 2 \mathrm{H}), 2.23(\mathrm{~s}, 3 \mathrm{H}), 2.34(\mathrm{~s}, 1 \mathrm{H}), 2.40(\mathrm{~s}$, $1 \mathrm{H}), 3.17-3.23(\mathrm{~m}, 3 \mathrm{H}), 3.62(\mathrm{~d}, J=10.0 \mathrm{~Hz}, 1 \mathrm{H}), 6.12(\mathrm{~d}, J=7.8 \mathrm{~Hz}, 1$ $\mathrm{H}), 6.34(\mathrm{~d}, J=7.5 \mathrm{~Hz}, 1 \mathrm{H}), 6.93(\mathrm{t}, J=7.65 \mathrm{~Hz}, 1 \mathrm{H})$.

${ }^{13} \mathrm{C}$ NMR $\left(125 \mathrm{MHz}, \mathrm{CDCl}_{3}\right): \delta=12.4,18.3,24.9,29.0,29.7,32.6,41.1$, 41.5, 50.2, 70.8, 102.3, 117.4, 127.7, 129.8, 133.9, 152.9 .

EI-MS: $m / z(\%)=227\left(\mathrm{M}^{\bullet+}, 100\right), 159(45), 198(24)$.

Anal. Calcd for $\mathrm{C}_{16} \mathrm{H}_{21} \mathrm{~N}$ : C, 84.53; H, 9.31; N, 6.16. Found: C, 84.20; H, 9.19; N, 5.93.

\section{9-Benzyl-5-methyl-2,3,4,4a,9,9a-hexahydro-1H-1,4-methano- carbazole (3c)}

Yield: $22 \mathrm{mg}$ (76\%); pale oil.

${ }^{1} \mathrm{H}$ NMR $\left(500 \mathrm{MHz}, \mathrm{CDCl}_{3}\right): \delta=1.15-1.20(\mathrm{~m}, 1 \mathrm{H}), 1.29-1.37(\mathrm{~m}, 2 \mathrm{H})$, 1.49-1.60 (m, $3 \mathrm{H}), 2.27$ (s, $4 \mathrm{H}), 2.46(\mathrm{~s}, 1 \mathrm{H}), 3.26(\mathrm{~d}, J=8.3 \mathrm{~Hz}, 1 \mathrm{H})$, $3.7(\mathrm{~d}, J=8.4 \mathrm{~Hz}, 1 \mathrm{H}), 4.34-4.42(\mathrm{~m}, 2 \mathrm{H}), 6.08(\mathrm{~d}, J=7.7 \mathrm{~Hz}, 1 \mathrm{H}), 6.4$ (d, J = 7.5 Hz, $1 \mathrm{H}), 6.90(\mathrm{t}, J=7.7 \mathrm{~Hz}, 1 \mathrm{H}), 7.30-7.35(\mathrm{~m}, 5 \mathrm{H})$.

${ }^{13} \mathrm{C}$ NMR $\left(125 \mathrm{MHz}, \mathrm{CDCl}_{3}\right): \delta=18.4,24.8,29.0,32.8,41.0,41.1,50.3$, 51.4, 72.2, 102.3, 117.9, 126.7, 127.0, 127.8, 128.4, 129.4, 134.0, 139.6, 153.5.

EI-MS: $m / z(\%)=289\left(\mathrm{M}^{\circ+}, 100\right), 221(51), 198$ (30).

Anal. Calcd for $\mathrm{C}_{21} \mathrm{H}_{23} \mathrm{~N}$ : C, 87.15; H, 8.01; N, 4.84. Found: C, 87.04; H, $7.89 ; \mathrm{N}, 4.51$.

\section{9-Butyl-5-methyl-2,3,4,4a,9,9a-hexahydro-1H-1,4-methanocarba- zole (3d)}

Yield: $18 \mathrm{mg}$ (72\%); yellow oil.

${ }^{1} \mathrm{H} \mathrm{NMR}\left(500 \mathrm{MHz}, \mathrm{CDCl}_{3}\right): \delta=0.96(\mathrm{t}, J=7.3 \mathrm{~Hz}, 3 \mathrm{H}), 1.11-1.16(\mathrm{~m}, 2$ H), 1.33-1.38 (m, $3 \mathrm{H}), 1.50-1.59(\mathrm{~m}, 5 \mathrm{H}), 2.23(\mathrm{~s}, 3 \mathrm{H}), 2.35(\mathrm{~s}, 1 \mathrm{H})$, 2.40 (s, $1 \mathrm{H}$ ), 3.12 (d, J = 7.6 Hz, $2 \mathrm{H}), 3.18$ (d, $J=8.4 \mathrm{~Hz}, 1 \mathrm{H}), 3.61$ (d, $J=8.4 \mathrm{~Hz}, 1 \mathrm{H}), 6.11(\mathrm{~d}, J=7.8 \mathrm{~Hz}, 1 \mathrm{H}), 6.34(\mathrm{~d}, J=7.5 \mathrm{~Hz}, 1 \mathrm{H}), 6.92(\mathrm{t}$, $J=7.6 \mathrm{~Hz}, 1 \mathrm{H})$.

${ }^{13} \mathrm{C}$ NMR $\left(125 \mathrm{MHz}, \mathrm{CDCl}_{3}\right): \delta=14.0,18.3,20.4,24.9,29.0,29.9,32.6$, 41.0, 41.4, 47.1, 50.2, 71.6, 102.1, 117.3, 127.7, 129.5, 133.8, 153.4 .

EI-MS: $m / z(\%)=255\left(\mathrm{M}^{*+}, 100\right), 187(54), 198$ (29).

Anal. Calcd for $\mathrm{C}_{18} \mathrm{H}_{25} \mathrm{~N}$ : C, 84.65; H, 9.87; N, 5.48. Found: C, 85.02; H, $10.00 ; \mathrm{N}, 5.71$.
5-Methyl-9-phenethyl-2,3,4,4a,9,9a-hexahydro-1H-1,4-methanocarbazole (3e)

Yield: $21 \mathrm{mg}$ (68\%); brown oil.

${ }^{1} \mathrm{H}$ NMR $\left(500 \mathrm{MHz}, \mathrm{CDCl}_{3}\right): \delta=1.15-1.24(\mathrm{~m}, 2 \mathrm{H}), 1.36-1.40(\mathrm{~m}, 1 \mathrm{H})$, 1.56-1.61 (m, $3 \mathrm{H}), 2.27(\mathrm{~s}, 3 \mathrm{H}), 2.31(\mathrm{~s}, 1 \mathrm{H}), 2.44(\mathrm{~s}, 1 \mathrm{H}), 2.83-2.96$ $(\mathrm{m}, 2 \mathrm{H}), 3.23(\mathrm{~d}, J=8.3 \mathrm{~Hz}, 1 \mathrm{H}), 3.41(\mathrm{t}, J=7.8 \mathrm{~Hz}, 2 \mathrm{H}), 3.65(\mathrm{~d}, J=8.2$ $\mathrm{Hz}, 1 \mathrm{H}), 6.18(\mathrm{~d}, J=7.8 \mathrm{~Hz}, 1 \mathrm{H}), 6.41(\mathrm{~d}, J=7.5 \mathrm{~Hz}, 1 \mathrm{H}), 6.98$ (t, $J=7.6$ $\mathrm{Hz}, 1 \mathrm{H}), 7.25-7.29(\mathrm{~m}, 3 \mathrm{H}), 7.35(\mathrm{t}, J=7.3 \mathrm{~Hz}, 2 \mathrm{H})$.

${ }^{13} \mathrm{C}$ NMR $\left(125 \mathrm{MHz}, \mathrm{CDCl}_{3}\right): \delta=18.4,24.9,29.0,32.7,34.0,41.1,41.4$, $49.5,50.3,71.8,102.2,117.8,126.1,127.8,128.4,128.8,129.7,134.0$, $140.0,152.9$.

EI-MS: $m / z(\%)=303\left(\mathrm{M}^{++}, 100\right), 235$ (57), 198 (41).

Anal. Calcd for $\mathrm{C}_{22} \mathrm{H}_{25} \mathrm{~N}$ : C, 87.08; H, 8.30; N, 4.62. Found: C, 86.76; H, $8.16 ; \mathrm{N}, 4.43$.

\section{9-Hexyl-5-methyl-2,3,4,4a,9,9a-hexahydro-1H-1,4-methanocarba- zole (3f)}

Yield: $25 \mathrm{mg}(88 \%)$; yellow oil.

${ }^{1} \mathrm{H} \mathrm{NMR}\left(500 \mathrm{MHz}, \mathrm{CDCl}_{3}\right): \delta=0.92-0.96(\mathrm{~m}, 4 \mathrm{H}), 1.33-1.38(\mathrm{~m}, 8 \mathrm{H})$, 1.54-1.63 (m, $5 \mathrm{H}), 2.24(\mathrm{~s}, 3 \mathrm{H}), 2.37(\mathrm{~s}, 1 \mathrm{H}), 2.42(\mathrm{~s}, 1 \mathrm{H}), 3.12(\mathrm{t}, J=$ $7.8 \mathrm{~Hz}, 2 \mathrm{H}), 3.20(\mathrm{~d}, J=8.3 \mathrm{~Hz}, 1 \mathrm{H}), 3.62(\mathrm{~d}, J=8.3 \mathrm{~Hz}, 1 \mathrm{H}), 6.12(\mathrm{~d}, J=$ $7.8 \mathrm{~Hz}, 1 \mathrm{H}), 6.35(\mathrm{~d}, J=6.5 \mathrm{~Hz}, 1 \mathrm{H}), 6.93(\mathrm{t}, J=7.6 \mathrm{~Hz}, 1 \mathrm{H})$.

${ }^{13} \mathrm{C}$ NMR $\left(125 \mathrm{MHz}, \mathrm{CDCl}_{3}\right): \delta=14.0,18.3,22.7,25.0,27.0,27.7,29.0$, 31.7, 32.6, 41.1, 41.4, 47.5, 50.2, 71.7, 102.1, 117.3, 127.7, 129.5, 133.8, 153.5 .

EI-MS: $m / z(\%)=283\left(\mathrm{M}^{++}, 100\right), 215$ (44), 198 (37).

Anal. Calcd for $\mathrm{C}_{20} \mathrm{H}_{29} \mathrm{~N}$ : C, 84.75; H, 10.31; N, 4.94. Found: C, 85.06; H, $10.43 ; \mathrm{N}, 5.17$

\section{9-Isopropyl-5-methyl-2,3,4,4a,9,9a-hexahydro-1H-1,4-methano- carbazole (3g)}

Yield: $20 \mathrm{mg}$ (81\%); pale oil.

${ }^{1} \mathrm{H} \mathrm{NMR}\left(500 \mathrm{MHz}, \mathrm{CDCl}_{3}\right): \delta=1.13(\mathrm{~d}, J=6.5 \mathrm{~Hz}, 3 \mathrm{H}), 1.22-1.25(\mathrm{~m}, 1$ $\mathrm{H}), 1.29(\mathrm{~d}, J=6.65 \mathrm{~Hz}, 4 \mathrm{H}), 1.32-1.36(\mathrm{~m}, 1 \mathrm{H}), 1.51-1.58(\mathrm{~m}, 3 \mathrm{H})$, $2.24(\mathrm{~s}, 3 \mathrm{H}), 2.29(\mathrm{~s}, 1 \mathrm{H}), 2.41(\mathrm{~s}, 1 \mathrm{H}), 3.20(\mathrm{~d}, J=8.5 \mathrm{~Hz}, 1 \mathrm{H}), 3.67$ (d, $J=8.5 \mathrm{~Hz}, 1 \mathrm{H}), 3.71-3.79(\mathrm{~m}, 1 \mathrm{H}), 6.20(\mathrm{~d}, J=7.8 \mathrm{~Hz}, 1 \mathrm{H}), 6.38(\mathrm{~d}, J=$ $7.5 \mathrm{~Hz}, 1 \mathrm{H}), 6.94(\mathrm{t}, J=7.7 \mathrm{~Hz}, 1 \mathrm{H})$.

${ }^{13} \mathrm{C}$ NMR $\left(125 \mathrm{MHz}, \mathrm{CDCl}_{3}\right): \delta=17.6,18.4,21.7,25.2,29.0,32.5,41.0$, 43.8, 47.4, 50.5, 66.8, 104.0, 117.8, 127.6, 130.4, 133.8, 152.9.

EI-MS: $m / z(\%)=241\left(\mathrm{M}^{\bullet+}, 100\right), 173$ (38), 198 (17).

Anal. Calcd for $\mathrm{C}_{17} \mathrm{H}_{23} \mathrm{~N}$ : C, 84.59; H, 9.60; N, 5.80. Found: C, 84.22; H, $9.47 ; \mathrm{N}, 5.52$.

\section{9-Isobutyl-5-methyl-2,3,4,4a,9,9a-hexahydro-1H-1,4-methano- carbazole (3h)}

Yield: $17 \mathrm{mg}$ (66\%); yellow oil.

${ }^{1} \mathrm{H}$ NMR $\left(500 \mathrm{MHz}, \mathrm{CDCl}_{3}\right): \delta=0.92(\mathrm{~d}, J=6.7 \mathrm{~Hz}, 3 \mathrm{H}), 0.96(\mathrm{~d}, J=6.7$ $\mathrm{Hz}, 3 \mathrm{H}), 1.09-1.16(\mathrm{~m}, 2 \mathrm{H}), 1.51-1.59(\mathrm{~m}, 4 \mathrm{H}), 1.94-2.05(\mathrm{~m}, 1 \mathrm{H})$, $2.23(\mathrm{~s}, 3 \mathrm{H}), 2.36-2.41(\mathrm{~m}, 2 \mathrm{H}), 2.83-2.96(\mathrm{~m}, 2 \mathrm{H}), 3.21(\mathrm{~d}, J=8.3 \mathrm{~Hz}$, $1 \mathrm{H}), 3.58(\mathrm{~d}, J=8.3 \mathrm{~Hz}, 1 \mathrm{H}), 6.11(\mathrm{~d}, J=7.8 \mathrm{~Hz}, 1 \mathrm{H}), 6.33(\mathrm{~d}, J=7.5 \mathrm{~Hz}$, $1 \mathrm{H}), 6.91(\mathrm{t}, J=7.7 \mathrm{~Hz}, 1 \mathrm{H})$.

${ }^{13} \mathrm{C}$ NMR $\left(125 \mathrm{MHz}, \mathrm{CDCl}_{3}\right): \delta=18.3,20.5,20.6,25.0,27.9,28.9,32.6$, $41.0,41.2,50.3,56.5,72.9,102.2,117.3,117.3,127.6,129.3,133.8$.

EI-MS: $m / z(\%)=255\left(\mathrm{M}^{+}, 100\right), 187(54), 198$ (51).

Anal. Calcd for $\mathrm{C}_{18} \mathrm{H}_{25} \mathrm{~N}$ : C, 84.65; H, 9.87; N, 5.48; Found: C, 84.98; H, 9.99; N, 5.70 . 
9-Cyclohexyl-5-methyl-2,3,4,4a,9,9a-hexahydro-1H-1,4-methanocarbazole (3i)

Yield: $16 \mathrm{mg}$ (57\%); yellow oil.

${ }^{1} \mathrm{H}$ NMR $\left(500 \mathrm{MHz}, \mathrm{CDCl}_{3}\right): \delta=1.07-1.13(\mathrm{~m}, 1 \mathrm{H}), 1.25-1.29(\mathrm{~m}, 3 \mathrm{H})$, 1.29-1.36 (m, $3 \mathrm{H}), 1.49-1.58$ (m, $4 \mathrm{H}), 1.64-1.76(\mathrm{~m}, 2 \mathrm{H}), 1.78-1.88$ (m, $2 \mathrm{H}), 1.94-2.02(\mathrm{~m}, 1 \mathrm{H}), 2.21(\mathrm{~s}, 3 \mathrm{H}), 2.26(\mathrm{~s}, 1 \mathrm{H}), 2.39(\mathrm{~s}, 1 \mathrm{H})$, $3.17(\mathrm{~d}, J=8.4 \mathrm{~Hz}, 1 \mathrm{H}), 3.21-3.31(\mathrm{~m}, 1 \mathrm{H}), 3.68(\mathrm{~d}, J=8.4 \mathrm{~Hz}, 1 \mathrm{H})$, $6.16(\mathrm{~d}, J=7.7 \mathrm{~Hz}, 1 \mathrm{H}), 6.33(\mathrm{~d}, J=7.3 \mathrm{~Hz}, 1 \mathrm{H}), 6.90(\mathrm{t}, J=7.6 \mathrm{~Hz}, 1 \mathrm{H})$. ${ }^{13} \mathrm{C}$ NMR $\left(125 \mathrm{MHz}, \mathrm{CDCl}_{3}\right): \delta=18.4,25.1,26.0,26.3,28.0,29.0,29.6$, $32.4,32.5,41.0,43.9,50.5,56.1,67.4,103.6,117.6,127.5,130.2$, $133.8,152.8$

EI-MS: $m / z(\%)=281\left(\mathrm{M}^{+}, 100\right), 213(37), 198(21)$.

Anal. Calcd for $\mathrm{C}_{20} \mathrm{H}_{27} \mathrm{~N}$ : C, 85.35; H, 9.67; N, 4.98. Found: C, 85.11; H, $9.53 ; \mathrm{N}, 4.84$.

\section{9-(2-Methoxyethyl)-5-methyl-2,3,4,4a,9,9a-hexahydro-1H-1,4-} methanocarbazole (3j)

Yield: $19 \mathrm{mg}$ (74\%); yellow oil.

${ }^{1} \mathrm{H}$ NMR $\left(500 \mathrm{MHz}, \mathrm{CDCl}_{3}\right): \delta=1.10-1.15(\mathrm{~m}, 1 \mathrm{H}), 1.27(\mathrm{~s}, 1 \mathrm{H}), 1.47-$ $1.52(\mathrm{~m}, 1 \mathrm{H}), 1.53-1.58(\mathrm{~m}, 2 \mathrm{H}), 2.22(\mathrm{~s}, 3 \mathrm{H}), 2.35-2.41(\mathrm{~m}, 2 \mathrm{H})$, $3.19(\mathrm{~d}, J=8.4 \mathrm{~Hz}, 1 \mathrm{H}), 3.25-3.32(\mathrm{~m}, 1 \mathrm{H}), 3.34-3.40(\mathrm{~m}, 4 \mathrm{H}), 3.47-$ $3.58(\mathrm{~m}, 2 \mathrm{H}), 3.66(\mathrm{~d}, J=8.3 \mathrm{~Hz}, 1 \mathrm{H}), 6.13(\mathrm{~d}, J=7.8 \mathrm{~Hz}, 1 \mathrm{H}), 6.35(\mathrm{~d}$, $J=7.5 \mathrm{~Hz}), 6.91(\mathrm{t}, J=7.6 \mathrm{~Hz}, 1 \mathrm{H})$.

${ }^{13} \mathrm{C}$ NMR $\left(125 \mathrm{MHz}, \mathrm{CDCl}_{3}\right): \delta=18.3,24.9,28.9,32.5,41.0,41.5,47.1$, $50.3,58.9,70.7,72.3,102.0,117.7,127.7,129.5,133.9,153.1$.

EI-MS: $m / z(\%)=257\left(\mathrm{M}^{\bullet+}, 100\right), 189(44), 198$ (31).

Anal. Calcd for $\mathrm{C}_{17} \mathrm{H}_{23} \mathrm{NO}$ : C, 79.33; H, 9.01; N, 5.44. Found: C, 79.65; H, 9.17; N, 5.68.

\section{9-Butyl-5-ethyl-2,3,4,4a,9,9a-hexahydro-1H-1,4-methanocarba- zole (3k)}

Yield: $15 \mathrm{mg}(54 \%)$; pale oil.

${ }^{1} \mathrm{H}$ NMR $\left(500 \mathrm{MHz}, \mathrm{CDCl}_{3}\right): \delta=0.99(\mathrm{t}, J=7.3 \mathrm{~Hz}, 3 \mathrm{H}), 1.13-1.20(\mathrm{~m}, 2$ $\mathrm{H}), 1.27(\mathrm{t}, J=7.7 \mathrm{~Hz}, 3 \mathrm{H}), 1.36-1.40(\mathrm{~m}, 2 \mathrm{H}), 1.53-1.65(\mathrm{~m}, 6 \mathrm{H})$, 2.36-2.42 (m, $2 \mathrm{H}), 2.55-2.65(\mathrm{~m}, 2 \mathrm{H}), 3.11-3.18(\mathrm{~m}, 2 \mathrm{H}), 3.24(\mathrm{~d}, J=$ $8.3 \mathrm{~Hz}, 1 \mathrm{H}), 3.63(\mathrm{~d}, J=8.4 \mathrm{~Hz}, 1 \mathrm{H}), 6.13(\mathrm{~d}, J=7.7 \mathrm{~Hz}, 1 \mathrm{H}), 6.42(\mathrm{~d}, J=$ $7.5 \mathrm{~Hz}, 1 \mathrm{H}), 7.00(\mathrm{t}, J=7.7 \mathrm{~Hz}, 1 \mathrm{H})$.

${ }^{13} \mathrm{C}$ NMR $\left(125 \mathrm{MHz}, \mathrm{CDCl}_{3}\right): \delta=14.1,14.7,20.5,24.9,25.1,29.1,30.0$, 32.6, 41.4, 41.9, 47.2, 49.9, 71.8, 102.0, 115.2, 127.9, 128.9, 140.0, 153.5.

EI-MS: $m / z(\%)=269\left(\mathrm{M}^{++}, 100\right), 201(55), 212(34)$.

Anal. Calcd for $\mathrm{C}_{19} \mathrm{H}_{27} \mathrm{~N}$ : C, 84.70; H, 10.10; N, 5.20. Found: C, 84.38; H, 9.96; N, 4.93.

\section{9-Benzyl-6,8-dichloro-2,3,4,4a,9,9a-hexahydro-1H-1,4-methano- carbazole (31)}

Yield: $30 \mathrm{mg}$ (89\%); orange oil.

${ }^{1} \mathrm{H} \mathrm{NMR}\left(500 \mathrm{MHz}, \mathrm{CDCl}_{3}\right): \delta=1.04-1.08(\mathrm{~m}, 1 \mathrm{H}), 1.14-1.17(\mathrm{~m}, 1 \mathrm{H})$, $1.28-1.32(\mathrm{~m}, 3 \mathrm{H}), 2.20(\mathrm{~d}, J=4.9 \mathrm{~Hz}, 1 \mathrm{H}), 2.25-2.28(\mathrm{~m}, 1 \mathrm{H}), 3.26-$ $3.29(\mathrm{~m}, 1 \mathrm{H}), 3.56-3.62(\mathrm{~m}, 2 \mathrm{H}), 4.71(\mathrm{~d}, J=16.2 \mathrm{~Hz}, 1 \mathrm{H}), 4.88(\mathrm{~d}, J=$ $16.2 \mathrm{~Hz}, 1 \mathrm{H}), 6.84-6.90$ (m, $1 \mathrm{H}), 6.95-7.0(\mathrm{~m}, 1 \mathrm{H}), 7.29-7.36$ (m, 5 $\mathrm{H})$.

${ }^{13} \mathrm{C}$ NMR $\left(125 \mathrm{MHz}, \mathrm{CDCl}_{3}\right): \delta=25.1,28.5,32.6,41.8,43.4,50.8,52.6$, 73.1, 113.2, 121.9, 123.3, 126.9, 127.4, 128.4, 128.9, 137.1, 139.6, 147.5.

EI-MS: $m / z(\%)=343\left(\mathrm{M}^{\bullet+}, 100\right), 275(52), 252(19)$.
Anal. Calcd for $\mathrm{C}_{20} \mathrm{H}_{19} \mathrm{Cl}_{2} \mathrm{~N}$ : C, 69.77; H, 5.56; N, 4.07. Found: C, 70.11; $\mathrm{H}, 5.67 ; \mathrm{N}, 4.29$.

9-Isobutyl-5-(trifluoromethyl)-2,3,4,4a,9,9a-hexahydro-1H-1,4methanocarbazole ( $3 \mathrm{~m})$

Yield: $19 \mathrm{mg}$ (61\%); pale oil.

${ }^{1} \mathrm{H}$ NMR $\left(500 \mathrm{MHz}, \mathrm{CDCl}_{3}\right): \delta=0.93(\mathrm{~d}, J=6.6 \mathrm{~Hz}, 3 \mathrm{H}), 0.97(\mathrm{~d}, J=6.6$ $\mathrm{Hz}, 3 \mathrm{H}), 1.28$ (s, $3 \mathrm{H}), 1.55-1.60(\mathrm{~m}, 2 \mathrm{H}), 1.97-2.0(\mathrm{~m}, 1 \mathrm{H}), 2.39-2.44$ (m, $1 \mathrm{H}), 2.51(\mathrm{~s}, 1 \mathrm{H}), 2.89-2.99(\mathrm{~m}, 3 \mathrm{H}), 3.44(\mathrm{~d}, J=8.8 \mathrm{~Hz}, 1 \mathrm{H}), 3.69$ $(\mathrm{d}, J=8.3 \mathrm{~Hz}, 1 \mathrm{H}), 6.34(\mathrm{~d}, J=8 \mathrm{~Hz}, 1 \mathrm{H}), 6.69(\mathrm{~d}, J=7.8 \mathrm{~Hz}, 1 \mathrm{H}), 7.05$ $(\mathrm{t}, J=7.9 \mathrm{~Hz}, 1 \mathrm{H})$.

${ }^{13} \mathrm{C}$ NMR $\left(125 \mathrm{MHz}, \mathrm{CDCl}_{3}\right): \delta=20.4,20.5,24.8,27.6,29.0,32.3,41.0$, $42.9,50.0,55.5,72.8,107.0,112.29(\mathrm{q}, J=5 \mathrm{~Hz}), 126.0,126.7(\mathrm{q}, J=$ $12.5 \mathrm{~Hz}), 127.5,128.1,155.0$.

EI-MS: $m / z(\%)=309\left(\mathrm{M}^{\bullet+}, 100\right), 241$ (59), 252 (33), 240 (14).

Anal. Calcd for $\mathrm{C}_{18} \mathrm{H}_{22} \mathrm{~F}_{3} \mathrm{~N}$ : C, 69.88; H, 7.17; N, 4.53. Found: C, 70.21; $\mathrm{H}, 7.28 ; \mathrm{N}, 4.69$.

\section{Acknowledgment}

We gratefully acknowledge financial support from the University of Tehran and Kharazmi University.

\section{Supporting Information}

Supporting information for this article is available online at https://doi.org/10.1055/s-0039-1707988.

\section{References}

(1) For reviews, see: (a) Liu, D.; Zhao, G.; Xiang, L. Eur. J. Org. Chem. 2010, 3975. (b) Silva, T. S.; Rodrigues, M. T. Jr.; Santos, H.; Zeoly, L. A.; Almeida, W. P.; Barcelos, R. C.; Gomes, R. C.; Fernandes, F. S.; Coelho, F. Tetrahedron 2019, 75, 2063.

(2) (a) Rosato, R. R.; Stephen, E. L.; Pannier, W. L. Toxicol. Appl. Pharmacol. 1976, 35, 107. (b) Bermudez, J.; Dabbs, S.; Joiner, K. A.; King, F. D. J. Med. Chem. 1990, 33, 1929. (c) Holsy, P. B.; Anthoni, U.; Christophersen, C.; Nielsen, P. H. J. Nat. Prod. 1994, 57, 997. (d) Triggle, D. J.; Mitchell, J. M.; Filler, R. CNS Drug Rev. 1998, 4, 87. (e) Austin, J. F.; Kim, S.-G.; Sinz, C. J.; Xiao, W.-J.; Macmillan, D. W. C. Proc. Natl. Acad. Sci. U. S. A. 2004, 101, 5482. (f) Gul, W.; Hamann, M. T. Life Sci. 2005, 78, 442. (g) Rode, M. A.; Rindhe, S. S.; Karale, B. J. Sreb. Chem. Soc. 2009, 74, 1377. (h) Ruiz-Sanchis, P.; Savina, S. A.; Albericio, F.; Álvarez, M. Chem. Eur. J. 2011, 17, 1388. (i) Annedi, S. C.; Ramnauth, J.; Maddaford, S. P.; Renton, P.; Rakhit, S.; Mladenova, G.; Dove, P.; Silverman, S.; Andrews, J. S.; Felice, M. D.; Porreca, F. J. Med. Chem. 2012, 55, 943.

(3) Zhang, H.; Boonsombat, J.; Padwa, A. Org. Lett. 2007, 9, 279.

(4) Bui, T.; Syed, S.; Barbas, C. F. III. J. Am. Chem. Soc. 2009, 131, 8758.

(5) Iyengar, R.; Schildknegt, K.; Morton, M.; Aube, J. J. Org. Chem. 2005, 70, 10645.

(6) Rakhit, A.; Hurley, M. E.; Tipnis, V.; Coleman, J.; Rommel, A.; Brunner, H. R. J. Clin. Pharmacol. 1986, 26, 156.

(7) Xiang, L.; Xing, D.; Wang, W.; Wang, R.; Ding, Y.; Du, L. Phytochemistry 2005, 66, 2595.

(8) For Buchwald-Hartwig amination, see: (a) Guram, A. S.; Rennels, R. A.; Buchwald, S. L. Angew. Chem. Int. Ed. 1995, 34, 1348. (b) Peat, A. J.; Buchwald, S. L. J. Am. Chem. Soc. 1996, 118 , 
1028. For representative indoline synthesis using BuchwaldHartwig amination reactions, see: (c) Yang, B. H.; Buchwald, S. L. Org. Lett. 1999, 1, 35. (d) Yamada, K.; Kubo, T.; Tokuyama, H.; Fukuyama, T. Synlett 2002, 231. (e) Omar-Amrani, R.; Thomas, A.; Brenner, E.; Schneider, R.; Fort, Y. Org. Lett. 2003, 5, 2311. (f) Omar-Amrani, R.; Schneider, R.; Fort, Y. Synthesis 2004, 2527. (g) Anderson, J. C.; Noble, A.; Tocher, D. A. J. Org. Chem. 2012, 77, 6703. (h) Harada, R.; Nishida, N.; Uchiito, S.; Onozaki, Y.; Kurono, N.; Senboku, H.; Masao, T.; Ohkuma, T.; Orito, K. Eur. J. Org. Chem. 2012, 366. (i) Ghorai, M. K.; Nanaji, Y. J. Org. Chem. 2013, 78, 3867. (j) Sirvent, J. A.; Foubelo, F.; Yus, M. J. Org. Chem. 2014, 79, 1356. (k) Sayyad, M.; Nanaji, Y.; Ghorai, M. J. Org. Chem. 2015, 80, 12659. (l) Presset, M.; Pignon, A.; Paul, J.; Le Gall, E.; Leonel, E.; Martens, T. J. Org. Chem. 2017, 82, 3302. (m) De Souza Fernandes, F.; Cormanich, R. A.; Zeoly, L. A.; Formiga, A. L. B.; Coelho, F. Eur. J. Org. Chem. 2018, 3211.

(9) (a) Li, J.-J.; Mei, T.-S.; Yu, J.-Q. Angew. Chem. Int. Ed. 2008, 47, 6452. (b) He, G.; Zhao, Y.; Zhang, S.; Lu, C.; Chen, G. J. Am. Chem. Soc. 2012, 134, 3. (c) Nadres, E. T.; Daugulis, O. J. Am. Chem. Soc. 2012, 134, 7. (d) He, G.; Lu, C.; Zhao, Y.; Nack, W. A.; Chen, G. Org. Lett. 2012, 14, 2944. (e) Mei, T.-S.; Leow, D.; Xiao, H.; Laforteza, B. N.; Yu, J.-Q. Org. Lett. 2013, 15, 3058. (f) Ye, X.; He, Z.; Ahmed, T.; Weise, K.; Akhmedov, N. G.; Petersen, J. L.; Shi, X. Chem. Sci. 2013, 4, 3712. (g) Wang, C.; Chen, C.; Zhang, J.; Han, J.; Wang, Q.; Guo, K.; Liu, P.; Guan, M.; Yao, Y.; Zhao, Y. Angew. Chem. Int. Ed. 2014, 53, 9884. (h) He, Y.-P.; Zhang, C.; Fan, M.; Wu, Z.; Ma, D. Org. Lett. 2015, 17, 496. (i) Takamatsu, K.; Hirano, K.; Satoh, T.; Miura, M. J. Org. Chem. 2015, 80, 3242. (j) Zheng, Y.; Song, W.; Zhu, Y.; Wei, B.; Xuan, L. Org. Biomol. Chem. 2018, 16, 2402. (k) Henry, M. C.; Senn, H. M.; Sutherland, A. J. Org. Chem. 2019, 84, 346.
(10) Kumar, G. S.; Singh, D.; Kumar, M.; Kapur, M.J. Org. Chem. 2018, 83, 3941.

(11) Zhao, D.; Vásquez-Céspedes, S.; Glorius, F. Angew. Chem. Int. Ed. 2015, 54, 1657.

(12) For reviews, see: (a) Catellani, M. Synlett 2003, 298. (b) Catellani, M. Top. Organomet. Chem. 2005, 14, 21. (c) Catellani, M.; Motti, E.; Della Ca', N. Acc. Chem. Res. 2008, 41, 1512. (d) Martins, A.; Mariampillai, B.; Lautens, M. Top. Curr. Chem. 2009, 292, 1. (e) Ye, J.; Lautens, M. Nat. Chem. 2015, 7, 863. (f) Della Ca', N.; Fontana, M.; Motti, E.; Catellani, M. Acc. Chem. Res. 2016, 49, 1389. (g) Liu, Z.-S.; Gao, Q.; Cheng, H.-G.; Zhou, Q. Chem. Eur. J. 2018, 24, 15461. (h) Zhao, K.; Ding, L.; Gu, Z. Synlett 2019, 30 , 129. (i) Wang, J.; Dong, G. Chem. Rev. 2019, 119, 7478.

(13) Zheng, H.; Zhu, Y.; Shi, Y. Angew. Chem. Int. Ed. 2014, 53, 11280.

(14) Liu, C.; Liang, Y.; Zheng, N.; Zhang, B.-S.; Feng, Y.; Bi, S.; Liang, Y.-M. Chem. Commun. 2018, 54, 3407.

(15) Wang, Z.; Li, P.; Fu, H.; Dai, Q.; Hu, C. Adv. Synth. Catal. 2019, 361, 192.

(16) Jafarpour, F.; Jalalimanesh, N.; Teimouri, M.; Shamsianpour, M. Chem. Commun. 2015, 51, 225.

(17) Annedi, S. C.; Ramnauth, J.; Maddaford, S. P.; Renton, P.; Rakhit, S.; Mladenova, G.; Dove, P.; Silverman, S.; Andrews, J. S.; Felice, M. D.; Porreca, F. J. Med. Chem. 2012, 55, 943.

(18) For related reviews on $\mathrm{C}-\mathrm{N}$ single bond cleavage reactions, see: (a) Ouyang, K.; Hao, W.; Zhang, W.-X.; Xi, Z. Chem. Rev. 2015, 115, 12045. (b) Desnoyer, A. N.; Love, J. A. Chem. Soc. Rev. 2017, 46, 197.

(19) Cardenas, D. J.; Martin-Matute, B.; Echavarren, A. M. J. Am. Chem. Soc. 2006, 128, 5033.

(20) Maestri, G.; Motti, E.; Della Ca', N.; Malacria, M.; Derat, E.; Catellani, M. J. Am. Chem. Soc. 2011, 133, 8574. 